\title{
CHARACTER EXPANSION OF KAC-MOODY CORRECTION FACTORS
}

\author{
KYU-HWAN LEE ${ }^{\star}$, DONGWEN LIU, AND THOMAS OLIVER ${ }^{\dagger}$
}

\begin{abstract}
A correction factor naturally arises in the theory of $p$-adic Kac-Moody groups. In this paper, we expand the correction factor into a sum of irreducible characters of the underlying Kac-Moody algebra. We derive a formula for the coefficients which lie in the ring of power series with integral coefficients. In the case that the Weyl group is a universal Coxeter group, we show that the coefficients are actually polynomials.
\end{abstract}

\section{INTRODUCTION}

Let $W$ be a Coxeter group, and consider its Poincaré series

$$
\chi(q):=\sum_{w \in W} q^{\ell(w)},
$$

where $q$ is an indeterminate and $\ell(w)$ is the length of $w$. R. Steinberg showed in [St68] that the series $\chi(q)$ represents a rational function in $q$. When $W$ is the Weyl group of an irreducible, reduced, finite root system $\Phi$, I.G. Macdonald [M72] found the following identity:

$$
\sum_{w \in W} \prod_{\alpha \in \Phi^{+}}\left(\frac{1-q e^{-w \alpha}}{1-e^{-w \alpha}}\right)=\chi(q),
$$

where $\Phi^{+}$is the set of positive roots and $e^{\beta}$ is a formal exponential associated to $\beta$ in the root lattice $Q$. Macdonald's identity reflects the geometry of the flag manifold.

A generalization of the left-hand side of (1.1) to a Kac-Moody root system $\Phi$ would be

$$
\mathcal{M}(q):=\sum_{w \in W} \prod_{\alpha \in \Phi^{+}}\left(\frac{1-q e^{-w \alpha}}{1-e^{-w \alpha}}\right)^{m(\alpha)},
$$

where $m(\alpha)$ is the multiplicity of $\alpha$. The identity (1.1) is no longer true for $\mathcal{M}(q)$, and so it is interesting to compute the correction factor ${ }^{1} \mathcal{M}(q) / \chi(q)$. Macdonald [M03] computed this quotient for the affine Kac-Moody case. The computation turns out to be equivalent to the Macdonald constant term conjecture [M82], which was proven by I. Cherednik in [Ch95].

The correction factor appears in the study of $p$-adic affine Kac-Moody groups, namely in the formal computation of Fourier coefficients of Eisenstein series and in the study of corresponding Hecke algebras. For example, it was shown by Braverman-Finkelberg-Kazhdan that this correction factor appears in the Gindikin-Karplevich formula for affine Kac-Moody groups [BFK] (see also

Date: May 11, 2021.

2010 Mathematics Subject Classification. Primary 17B22, 17B67; Secondary 05E10.

${ }^{\star}$ This work was partially supported by a grant from the Simons Foundation (\#318706).

${ }^{\dagger}$ This article arises from research funded by the John Fell Oxford University Press Research Fund.

${ }^{1}$ A slight modification of this quotient, denoted by $\mathfrak{m}$, is what Macdonald called the constant term in the affine case and is also called the "correction factor" in the literature (see (2.10) for a precise definition). 
[BGKP, BKP, GR14, BPGR16]). The correction factor in the general case was studied by MuthiahPuskas-Whitehead $[\mathrm{MPW}$. They encoded the data of the correction factor into a collection of polynomials indexed by positive imaginary roots and derived formulas for these polynomials.

In this paper, we study the correction factor $\mathcal{M}(q) / \chi(q)$ for arbitrary Kac-Moody root systems, which we write as a sum of characters $\operatorname{ch}(L(\lambda))$ of integrable irreducible representations $L(\lambda)$ of the Kac-Moody algebra $\mathfrak{g}$ with root system $\Phi$. As the first main result of this paper, we prove that the sum is supported on $\lambda \in P^{+} \cap Q_{\mathrm{im}}^{-}$, where $P^{+}$is the set of dominant integral weights and $Q_{\mathrm{im}}^{-}$ is the cone generated by negative imaginary roots. More precisely, we obtain

Theorem 1.1. Given a Kac-Moody algebra $\mathfrak{g}$, let $P^{+}$denote its set of dominant integral weights and $Q_{\mathrm{im}}^{-}$its negative imaginary root cone. Then there are $d_{\lambda} \in \mathbb{Z}[[q]], \lambda \in P^{+} \cap Q_{\mathrm{im}}^{-}$, such that

$$
\mathcal{M}(q) / \chi(q)=\sum_{\lambda \in P^{+} \cap Q_{\mathrm{im}}^{-}} d_{\lambda} \operatorname{ch}(L(\lambda)) .
$$

Actually, we prove this result for any $W$-invariant functions with support in the negative root cone $Q^{-}$(see Theorem 2.13). We recover (1.1) as an immediate consequence, since $P^{+} \cap Q_{\text {im }}^{-}=\{0\}$ for finite root systems. This result also explains why the known formulas in the affine case only involve imaginary roots.

The coefficients $d_{\lambda}$ are related to the function $H(\mu ; q), \mu \in Q$, which was introduced by Kim and Lee [KL11, KL12] in a study of $p$-adic integrals using canonical/crystal bases from the context of Weyl group multiple Dirichlet series ([Bu12] for a survey). See Definition 3.1 for the definition of $H(\mu ; q)$. We prove the following formula (Theorem 3.5):

$$
\chi(q) d_{\lambda}=\sum_{w \in W}(-1)^{\ell(w)} H(-w \circ \lambda ; q),
$$

where $w \circ \lambda:=w(\lambda+\rho)-\rho$ with a Weyl vector $\rho$.

Using (1.3), one can compute $d_{\lambda}$ explicitly. In particular, in the rank 2 hyperbolic case, we observe that they are actually polynomials in $q$. Generalizing this observation, we prove that $d_{\lambda}$ are always polynomials when $W$ is a universal Coxeter group, or equivalently, when $a_{i j} a_{j i} \geq 4$ for all $i, j \in I$ with the generalized Cartan matrix $A=\left(a_{i j}\right)_{i, j \in I}$ of $\mathfrak{g}$. Formally, we obtain

Theorem 1.2. Assume that the Weyl group $W$ of $\mathfrak{g}$ is a universal Coxeter group. Then we have $d_{\lambda} \in \mathbb{Z}[q]$ for all $\lambda \in P^{+} \cap Q_{\mathrm{im}}^{-}$.

It would be very interesting to see if $d_{\lambda}$ are polynomials for arbitrary Kac-Moody root systems. We expect that these coefficients carry important combinatorial, representation-theoretic information, which is yet to be revealed. We hope that we can investigate these issues in the near future.

The main text proceeds as follows. In Section 2 we review standard background material and construct a large ring containing $\mathcal{M}(q)$ equipped with a $W$-action. We conclude with the statement that $W$-invariant elements admit a character expansion, which applies in particular to $\mathcal{M}(q)$. In Section 3 we compute the character coefficients in terms of the function $H$. Though the formula deduced involves an infinite sum, it exhibits a large amount of cancellation and in Section 4 we show that it is in fact a polynomial when $W$ is a universal Coxeter group. In the Appendix, we give compute the coefficients for certain small imaginary roots of a rank 2 hyperbolic Kac-Moody algebra. 
Acknowledgments. We thank Dinakar Muthiah, Anna Puskás and Ian Whitehead for helpful discussions, and are grateful to the anonymous referee for helpful comments.

\section{Existence of CHARACTER COEFFiCiEnts}

We will use the conventions and terminology of [Ka90]. Let $I=\{1, \cdots, n\}$ and let $A$ be a generalized Cartan matrix with realisation $\left(\mathfrak{h}, \Pi, \Pi^{\vee}\right)$. In particular, the elements of the set $\Pi=\left\{\alpha_{1}, \ldots, \alpha_{n}\right\} \subset \mathfrak{h}^{*}\left(\right.$ resp. $\left.\Pi^{\vee}=\left\{\alpha_{1}^{\vee}, \ldots, \alpha_{n}^{\vee}\right\} \subset \mathfrak{h}\right)$ are the simple roots (resp. simple coroots). The root lattice $Q$ (resp. positive root cone $Q^{+}$) is the $\mathbb{Z}$-span (resp. $\mathbb{Z}_{\geq 0^{-}}$span) of $\Pi$. We set $Q^{-}=-Q^{+}$. A partial order $\geq$on $\mathfrak{h}^{*}$ is defined by $\mu \geq \nu$ if $\mu-\nu \in Q^{+}$. We say $\alpha \in \mathfrak{h}^{*}$ is positive (resp. negative) if $\alpha>0$ (resp. $\alpha<0$ ).

Let $\mathfrak{g}$ be the Kac-Moody algebra associated to $A$, which admits the root space decomposition $\mathfrak{g}=\oplus_{\alpha \in Q} \mathfrak{g}_{\alpha}$, with $\mathfrak{g}_{0}=\mathfrak{h}$. Given $\alpha \in Q$, its multiplicity $m(\alpha)$ is the dimension of the vector space $\mathfrak{g}_{\alpha}$. A non-zero $\alpha \in Q$ is a root if $m(\alpha) \neq 0$. We will denote the set of roots by $\Phi$, and the set of positive (resp. negative) roots by $\Phi^{+}$(resp. $\Phi^{-}$).

Let $W$ denote the Weyl group of $\mathfrak{g}$, which is the subgroup of Aut $\left(\mathfrak{h}^{*}\right)$ generated by the simple reflections $s_{i}, i \in I$. A root $\alpha \in \Phi$ is called real if there is $w \in W$ such that $w \alpha$ is a simple root. A root that is not real is called imaginary. If $\alpha$ is real, then $m(\alpha)=1$. The set of real (resp. imaginary) roots is denoted by $\Phi_{\text {re }}\left(\right.$ resp. $\left.\Phi_{\text {im }}\right)$, and the set of positive real (resp. positive imaginary) roots is denoted by $\Phi_{\mathrm{re}}^{+}\left(\operatorname{resp} . \Phi_{\mathrm{im}}^{+}\right)$.

Let $q$ denote a formal variable, and let $\mathbb{Z}[[q]]$ be the ring of power series in $q$ with integer coefficients. Recall that $f(q) \in \mathbb{Z}[[q]]$ is invertible if and only if the constant term $f(0)$ of $f$ is equal to \pm 1 . The inverse of a unit in $\mathbb{Z}[[q]]$ will be written as a fraction whenever it is convenient. For example, we write

$$
\frac{1}{1-q}=1+q+q^{2}+\cdots
$$

Example 2.1. The Poincaré series of the Weyl group $W$ is defined as follows:

$$
\chi(q)=\sum_{w \in W} q^{\ell(w)} \in \mathbb{Z}[[q]],
$$

where the length $\ell(w)$ of $w \in W$ is the minimal $\ell$ such that $w=s_{i_{1}} \cdots s_{i_{\ell}}$ is a product of simple reflections. As the only word of length 0 is the identity element, the constant term of $\chi(q)$ is 1 . Thus, $\chi(q) \in \mathbb{Z}[[q]]^{\times}$.

Notation. To each $\lambda \in \mathfrak{h}^{*}$, we associate a formal exponential denoted by $e^{\lambda}$, and define $e^{\lambda} e^{\mu}=$ $e^{\lambda+\mu}$ for $\lambda, \mu \in \mathfrak{h}^{*}$. Let $\mathbb{Z}((q))$ denote the ring of Laurent series with integral coefficients, and let $R$ be a subring of $\mathbb{Z}((q))$. We denote by $\mathcal{S}(R)$ the additive group of formal sums $\sum_{\lambda \in \mathfrak{h}^{*}} a_{\lambda} e^{\lambda}$ with $a_{\lambda} \in R$ for all $\lambda \in \mathfrak{h}^{*}$.

Definition 2.2. The support of a formal sum $\sum_{\lambda \in \mathfrak{h}^{*}} a_{\lambda} e^{\lambda} \in \mathcal{S}(R)$ is the set of $\lambda \in \mathfrak{h}^{*}$ such that $a_{\lambda} \neq 0$.

If $f=\sum_{\lambda \in Q} a_{\lambda} e^{\lambda}$ is a unit of $\mathcal{S}(R)$ and has support in a translate of $Q^{-}$, then $f$ has a unique product expansion as in [MPW, Proposition 2.2]:

$$
\sum_{\lambda \in Q} a_{\lambda} e^{\lambda}=u e^{\lambda_{0}} \prod_{\lambda \in Q^{-} \backslash\{0\}} \prod_{n \in \mathbb{Z}}\left(1-q^{n} e^{\lambda}\right)^{m(\lambda, n)}
$$

for some $u \in R^{\times}, \lambda_{0} \in Q$ and $m(\lambda, n) \in \mathbb{Z}$ such that, for every $\lambda$, the set $\{n \in \mathbb{Z}: m(\lambda, n) \neq 0\}$ is bounded below. 
Definition 2.3 (Section 2.3 in [MPW]). A product of the form (2.2) is called a good product with coefficients in $R$ if all $\lambda$ appearing in its factors are multiples of roots $\alpha \in \Phi$, and the set of factors corresponding to any real root $\alpha \in \Phi_{\text {re }}$ is finite. We will denote by $\mathcal{G}(R)$ the multiplicative group of good products with coefficients in $R$.

An element of $\mathcal{G}(R)$ expands to a formal sum in $\mathcal{S}(R)$ by definition. The notion of a good product is introduced, in part, to define the action of $W$ as below.

Definition 2.4. We define an action of $W$ on $\mathcal{G}(R)$ by extending the following action on the factors of (2.2) multiplicatively:

$$
w\left(1-q^{n} e^{\lambda}\right)= \begin{cases}1-q^{n} e^{w(\lambda)}, & w(\lambda)<0, \\ \left(-q^{n} e^{w(\lambda)}\right)\left(1-q^{-n} e^{-w(\lambda)}\right), & w(\lambda)>0,\end{cases}
$$

for $w \in W$. Given $f \in \mathcal{G}(R)$, we will sometimes write $f^{w}=w(f)$. We will denote by $\mathcal{G}^{W}(R)$ the ring of $W$-invariant elements of $\mathcal{G}(R)$.

Define the negative imaginary cone $Q_{\mathrm{im}}^{-}$to be the cone generated by negative imaginary roots. Then we have $Q_{\mathrm{im}}^{-}=\bigcap_{w \in W} w\left(Q^{-}\right)$. Thus if $f \in \mathcal{G}^{W}(R)$ is supported on $Q^{-}$, then it is in fact supported on $Q_{\mathrm{im}}^{-}$. It was noted in [MPW] that, for $w \in W$ and $f=\sum_{\lambda \in \mathfrak{h}^{*}} a_{\lambda} e^{\lambda} \in \mathcal{G}(R)$, we have

$$
w(f)=\sum_{\lambda \in \mathfrak{h}^{*}} a_{\lambda} e^{w \lambda}
$$

Remark 2.5. The set of $f \in \mathcal{S}(R)$ supported on $Q^{-}$is not closed under the action of $W$ defined by $(2.4)$, but $\mathcal{G}(R)$ is.

The basic good product in this paper is

$$
\Delta:=\prod_{\alpha \in \Phi^{+}}\left(\frac{1-q e^{-\alpha}}{1-e^{-\alpha}}\right)^{m(\alpha)} .
$$

Here $\frac{1-q e^{-\alpha}}{1-e^{-\alpha}}=1+\sum_{n \geq 1}(1-q) e^{-n \alpha}$, and it is clear that $\Delta \in \mathcal{G}(\mathbb{Z}[q])$.

Since $m(\alpha)=1$ for $\alpha \in \Phi_{\text {re }}^{+}$, we set

$$
\Delta_{\mathrm{re}}:=\prod_{\alpha \in \Phi_{\mathrm{re}}^{+}}\left(\frac{1-q e^{-\alpha}}{1-e^{-\alpha}}\right), \quad \Delta_{\mathrm{im}}:=\prod_{\alpha \in \Phi_{\mathrm{im}}^{+}}\left(\frac{1-q e^{-\alpha}}{1-e^{-\alpha}}\right)^{m(\alpha)}
$$

so that we have

$$
\Delta=\Delta_{\mathrm{re}} \Delta_{\mathrm{im}}
$$

Finally, define

$$
\mathcal{M}(q):=\sum_{w \in W} \Delta^{w}
$$

Clearly, $\mathcal{M}(q)$ is $W$-invariant since it is the sum of $W$-action on $\Delta$.

Lemma 2.6. The formal sum $\mathcal{M}(q)$ is a $W$-invariant good product with coefficients in $\mathbb{Z}[[q]]$, i.e. $\mathcal{M}(q) \in \mathcal{G}^{W}(\mathbb{Z}[[q]])$. Moreover, $\mathcal{M}(q)$ is supported on $Q_{\mathrm{im}}^{-}$and has the constant term equal to $\chi(q)$. 
Proof. Since the set $\Phi_{\mathrm{im}}^{+}$is $W$-invariant, and $m(w \alpha)=m(\alpha)$ for $w \in W$ and $\alpha \in \Phi$, we have $\Delta_{\mathrm{im}}^{w}=\Delta_{\mathrm{im}}$. It follows that $\Delta^{w}=\left(\Delta_{\mathrm{re}} \Delta_{\mathrm{im}}\right)^{w}=\Delta_{\mathrm{re}}^{w} \Delta_{\mathrm{im}}$. By Definition 2.4, we have

$$
w\left(\frac{1-q e^{-\alpha}}{1-e^{-\alpha}}\right)= \begin{cases}\frac{1-q e^{-w(\alpha)}}{1-e^{-w(\alpha)}} & \text { if } w(\alpha)>0, \\ \frac{q e^{-w(\alpha)}\left(1-q^{-1} e^{w(\alpha)}\right)}{e^{-w(\alpha)}\left(1-e^{w(\alpha)}\right)}=\frac{q\left(1-q^{-1} e^{w(\alpha)}\right)}{1-e^{w(\alpha)}} & \text { if } w(\alpha)<0,\end{cases}
$$

for $w \in W$ and $\alpha \in \Phi_{\mathrm{re}}^{+}$. One can immediately see that the sum $\mathcal{M}(q)$ is supported on $Q^{-}$. Since

$$
\frac{1-q e^{-\alpha}}{1-e^{-\alpha}}=1+\sum_{n \geq 1}(1-q) e^{-n \alpha} \text { and } \frac{q\left(1-q^{-1} e^{-\alpha}\right)}{1-e^{-\alpha}}=\frac{q-e^{-\alpha}}{1-e^{-\alpha}}=q-\sum_{n \geq 1}(1-q) e^{-n \alpha},
$$

we see that $\Delta^{w}$ is a good product with coefficients in $\mathbb{Z}[q]$, i.e. $\Delta^{w} \in \mathcal{G}(\mathbb{Z}[q])$.

Now we check that the coefficient of $e^{-\beta}$ in $\mathcal{M}(q)=\sum_{w \in W} \Delta^{w}$ is an element of $\mathbb{Z}[[q]]$ for $\beta \in Q^{+}$. For $w \in W$, define

$$
\Phi(w):=\left\{\alpha \in \Phi_{\mathrm{re}}^{+} \mid w(\alpha)<0\right\}=\Phi^{+} \cap w^{-1} \Phi^{-} .
$$

It is well-known that $|\Phi(w)|=\ell(w)$. Thus we have

$$
\begin{aligned}
\Delta_{\mathrm{re}}^{w} & =\prod_{\alpha \in \Phi\left(w^{-1}\right)}\left(\frac{q-e^{-\alpha}}{1-e^{-\alpha}}\right) \prod_{\alpha \in \Phi_{\mathrm{re}}^{+} \backslash \Phi\left(w^{-1}\right)}\left(\frac{1-q e^{-\alpha}}{1-e^{-\alpha}}\right) \\
& =q^{\ell(w)} \prod_{\alpha \in \Phi\left(w^{-1}\right)}\left(\frac{1-q^{-1} e^{-\alpha}}{1-e^{-\alpha}}\right) \prod_{\alpha \in \Phi_{\mathrm{re}}^{+} \backslash \Phi\left(w^{-1}\right)}\left(\frac{1-q e^{-\alpha}}{1-e^{-\alpha}}\right) .
\end{aligned}
$$

For $\beta \in Q^{+}$, the coefficient of $e^{-\beta}$ in $\Delta^{w}=\Delta_{\mathrm{re}}^{w} \Delta_{\text {im }}$, a priori an element in $\mathbb{Z}[[q]]$, is of the form

$$
q^{\ell(w)} p_{\beta, w}
$$

for some $p_{\beta, w} \in \mathbb{Z}((q))$. Recall the height of $\beta=\sum_{i=1}^{n} m_{i} \alpha_{i} \in Q^{+}, m_{i} \geq 0$, is defined to be

$$
\operatorname{ht}(\beta):=\sum_{i=1}^{n} m_{i} .
$$

It is easy to observe from (2.8) and (2.9) the crude estimate that the degrees of $p_{\beta, w}$ in $q^{-1}$ and $q$ are both bounded by $\operatorname{ht}(\beta)$. Thus we have $p_{\beta, w} \in \mathbb{Z}\left[q, q^{-1}\right]$. Moreover $q^{m}$ appears in $q^{\ell(w)} p_{\beta, w}$ only if $\ell(w) \leq m+\operatorname{ht}(\beta)$. Since there are only finitely many $w \in W$ of a given length, we see that

$$
\mathcal{M}(q)=\sum_{w \in W} \Delta^{w}=\sum_{\beta \in Q^{+}}\left(\sum_{w \in W} q^{\ell(w)} p_{\beta, w}\right) e^{-\beta}
$$

with the coefficient of $e^{-\beta}$ given by a well-defined sum

$$
\sum_{w \in W} q^{\ell(w)} p_{\beta, w} \in \mathbb{Z}[[q]]
$$

In particular, when $\beta=0$, we have $p_{0, w}=1$ for all $w \in W$ and the constant term of $\mathcal{M}(q)$ is equal to $\sum_{w \in W} q^{\ell(w)}=\chi(q)$.

We have already seen that $\mathcal{M}(q)$ is supported on $Q^{-}$at the beginning of the proof. Since $\mathcal{M}(q)$ is also $W$-invariant, it is supported on $Q_{\mathrm{im}}^{-}$. (See the paragraph after Definition 2.4.) 
Using [MPW, Proposition 2.2], we may write $\mathcal{M}(q)$ as a product of the form (2.2) with $\lambda_{0}=0$. Since $\mathcal{M}(q)$ is supported on $Q_{\text {im }}^{-}$, no factor corresponding to a real root arises in the product and hence $\mathcal{M}(q)$ is a good product.

Remark 2.7. (1) We have the following identity in $\mathcal{G}^{W}(\mathbb{Z}[[q]])$ :

$$
\mathfrak{m} \mathcal{M}(q)=\Delta_{\operatorname{im}} \chi(q),
$$

where $\mathfrak{m}$ is as defined in [MPW, equation (3.5)]. Each of $\mathfrak{m}^{-1}, \Delta_{\text {im }}$ and $\mathcal{M}(q)$ expands to a formal sum supported on $Q_{\mathrm{im}}^{-}$.

(2) In the paper [BPGR19], it was pointed out that $\mathcal{M}(q)$ is not an element of $\mathcal{G}^{W}\left(\mathbb{Z}\left[q, q^{-1}\right]\right)$ but an element of $\mathcal{G}^{W}(\mathbb{Z}((q)))$. As a refinement, Lemma 2.6 shows that $\mathcal{M}(q) \in \mathcal{G}^{W}(\mathbb{Z}[[q]])$.

Now we move on to study a character expansion of an element in $\mathcal{G}^{W}(\mathbb{Z}[[q]])$.

Definition 2.8. Fix a Weyl vector $\rho \in \mathfrak{h}^{*}$, i.e. a vector satisfying $\rho\left(\alpha_{i}^{\vee}\right)=1$, for all $i \in I$. The circle action $^{2}$ of $W$ on $\mathfrak{h}^{*}$ is defined by

$$
w \circ \lambda=w(\lambda+\rho)-\rho .
$$

Example 2.9. We have

$$
w \circ 0=w \rho-\rho,
$$

which can be written as a sum of negative roots. Indeed, one has

$$
\rho-w \rho=\sum_{\alpha \in \Phi\left(w^{-1}\right)} \alpha,
$$

where, for $w \in W$,

$$
\Phi(w):=\Phi^{+} \cap w^{-1} \Phi^{-} .
$$

Denote by $P$ the weight lattice of $\mathfrak{g}$, and by $P^{+} \subset P$ the subset of dominant integral weights. For $\lambda \in P$, define

$$
\pi^{\lambda}:=\frac{\sum_{w \in W}(-1)^{\ell(w)} e^{w(\lambda+\rho)}}{\sum_{w \in W}(-1)^{\ell(w)} e^{w \rho}} .
$$

Recall the denominator identity

$$
\sum_{w \in W}(-1)^{\ell(w)} e^{w \rho-\rho}=\prod_{\alpha \in \Phi^{+}}\left(1-e^{-\alpha}\right)^{m(\alpha)} .
$$

For $\lambda \in P$, define

$$
\xi^{\lambda}:=\sum_{w \in W}(-1)^{\ell(w)} e^{w \circ \lambda}
$$

\section{Lemma 2.10.}

(1) For $w \in W$, we have

$$
w\left(\prod_{\alpha \in \Phi^{+}}\left(1-e^{-\alpha}\right)^{m(\alpha)}\right)=(-1)^{\ell(w)} e^{\rho-w \rho} \prod_{\alpha \in \Phi^{+}}\left(1-e^{-\alpha}\right)^{m(\alpha)} .
$$

\footnotetext{
${ }^{2}$ This action is slightly different to the action with the same notation in [KL12].
} 
(2) For $\lambda \in P$ and $w \in W$, we have

$$
\begin{aligned}
& \xi^{\lambda}=(-1)^{\ell(w)} \xi^{w \circ \lambda} \\
& \pi^{\lambda}=(-1)^{\ell(w)} \pi^{w \circ \lambda}
\end{aligned}
$$

Proof. (1) From the denominator identity (2.15), we have

$$
\begin{aligned}
w\left(\prod_{\alpha \in \Phi^{+}}\left(1-e^{-\alpha}\right)^{m(\alpha)}\right) & =\sum_{w_{1} \in W}(-1)^{\ell\left(w_{1}\right)} e^{w w_{1} \rho-w \rho} \\
& =\sum_{w_{1} \in W}(-1)^{\ell(w)+\ell\left(w w_{1}\right)} e^{w w_{1} \rho-\rho} e^{\rho-w \rho} \\
& =(-1)^{\ell(w)} e^{\rho-w \rho} \sum_{w_{1} \in W}(-1)^{\ell\left(w w_{1}\right)} e^{w w_{1} \rho-\rho} \\
& =(-1)^{\ell(w)} e^{\rho-w \rho} \prod_{\alpha \in \Phi^{+}}\left(1-e^{-\alpha}\right)^{m(\alpha)} .
\end{aligned}
$$

(2) Let $w \circ \lambda=\mu$. Then $w(\lambda+\rho)=\mu+\rho$. Now we have

$$
\begin{aligned}
\sum_{w_{1} \in W}(-1)^{\ell\left(w_{1}\right)} e^{w_{1}(\lambda+\rho)} & =\sum_{w_{1} \in W}(-1)^{\ell(w)+\ell\left(w_{1} w^{-1}\right)} e^{w_{1} w^{-1} w(\lambda+\rho)} \\
& =(-1)^{\ell(w)} \sum_{w_{1} \in W}(-1)^{\ell\left(w_{1} w^{-1}\right)} e^{w_{1} w^{-1}(\mu+\rho)} .
\end{aligned}
$$

Multiplying both sides by $e^{-\rho}$, we get $\xi^{\lambda}=(-1)^{\ell(w)} \xi^{\mu}=(-1)^{\ell(w)} \xi^{w \circ \lambda}$. Dividing both sides by $\sum_{w_{1} \in W}(-1)^{\ell\left(w_{1}\right)} e^{w_{1} \rho-\rho}$, we obtain $\pi^{\lambda}=(-1)^{\ell(w)} \pi^{w \circ \lambda}$.

Consider the following subset of $Q^{-}$:

$$
Q^{\prime}:=\bigcap_{w \in W} w \circ Q^{-} .
$$

The Weyl group $W$ acts on $Q^{\prime}$ by the circle action, and so $Q_{\mathrm{im}}^{-} \subset Q^{\prime}$.

Lemma 2.11. Assume that $\lambda \in Q^{\prime}$. Then the following hold.

(1) There exists a unique $\mu \in Q^{-}$and $v \in W$ such that $\mu+\rho \in P^{+}$and $v \circ \lambda=\mu$.

(2) The stabilizer subgroup

$$
W_{\lambda}^{\circ}:=\{w \in W: w \circ \lambda=\lambda\}
$$

is generated by reflections in $W$.

(3) If $\lambda \in P^{+} \cap Q^{-}$, then $W_{\lambda}^{\circ}=\{1\}$.

Proof. Write $\lambda=\sum_{i} m_{i} \alpha_{i}$ with $m_{i} \leq 0$ for all $i$. If $\lambda+\rho \in P^{+}$, there is nothing to prove. If not, there exists $j$ such that $\lambda\left(\alpha_{j}^{\vee}\right) \leq-2$. We have

$$
s_{j} \circ \lambda=s_{j}(\lambda+\rho)-\rho=\lambda-\left(\lambda\left(\alpha_{j}^{\vee}\right)+1\right) \alpha_{j} \in Q^{-} .
$$

Since $\lambda\left(\alpha_{j}^{\vee}\right)+1<0$, we have $m_{j}<m_{j}-\left(\lambda\left(\alpha_{j}^{\vee}\right)+1\right) \leq 0$. If $\left(s_{j} \circ \lambda\right)+\rho$ is in $P^{+}$, we are done. Otherwise, repeat the process with replacing $\lambda$ with $s_{j} \circ \lambda$. Since the coefficients are increasing and bounded above by 0 , this process must end. 
Assume that $\lambda+\rho \in P^{+}$. Suppose that $w \circ \lambda=\mu$ and $\mu+\rho \in P^{+}$for $w=s_{i_{1}} s_{i_{2}} \cdots s_{i_{\ell}} \neq 1$, a reduced expression. Then we have $w(\lambda+\rho)=\mu+\rho$. Since $(\lambda+\rho)\left(\alpha_{i_{\ell}}^{\vee}\right) \geq 0$, we have $(\mu+\rho)\left(w\left(\alpha_{i_{\ell}}^{\vee}\right)\right) \geq$ 0 . Since $w=s_{i_{1}} s_{i_{2}} \cdots s_{i_{\ell}}$ is a reduced expression, we get $w\left(\alpha_{i_{\ell}}^{\vee}\right)<0$, and $(\mu+\rho)\left(w\left(\alpha_{i_{\ell}}^{\vee}\right)\right) \leq 0$. Thus $(\mu+\rho)\left(w\left(\alpha_{i_{\ell}}^{\vee}\right)\right)=0$ and $(\lambda+\rho)\left(\alpha_{i_{\ell}}^{\vee}\right)=0$. Hence $s_{i_{\ell}}(\lambda+\rho)=\lambda+\rho$ and $s_{i_{\ell}} \circ \lambda=\lambda$. By induction, we obtain $\mu=\lambda$, which completes a proof of (1). We have also shown that the subgroup $W_{\lambda}^{\circ}$ is generated by simple reflections for $\lambda+\rho \in P^{+}$.

Assume that $\lambda \in P^{+} \cap Q^{-}$, and suppose that $w \circ \lambda=\lambda$ for $w=s_{i_{1}} s_{i_{2}} \cdots s_{i_{\ell}} \neq 1$, a reduced expression. Then $(\lambda+\rho)\left(\alpha_{i_{\ell}}^{\vee}\right)>0$ and the above argument leads to a contradiction. Thus we must have $w=1$. This proves $(3)$.

Now assume that $\lambda \in Q^{\prime}$. By Lemma 2.11(1), there exists $v \in W$ such that $v \circ \lambda+\rho \in P^{+}$. Then $W_{v \circ \lambda}^{\circ}$ is generated by simple reflections $s_{i}$. Hence $W_{\lambda}^{\circ}$ is generated by $v^{-1} s_{i} v$, which are reflections. This completes a proof of (2).

Lemma 2.12. Assume that $\lambda \in Q^{\prime}$. The series $\xi^{\lambda} \in \mathcal{G}(\mathbb{Z})$ defined in (2.16) is non-zero if and only if the stabilizer subgroup $W_{\lambda}^{\circ}$ of $\lambda$ under the circle action is trivial.

Proof. Suppose that $\xi^{\lambda}=0$. Then the term $e^{\lambda}$ cancels with $(-1)^{\ell(w)} e^{w \circ \lambda}$ for some $w \neq 1$. In particular, $\lambda=w \circ \lambda$, and the stabilizer subgroup $W_{\lambda}^{\circ}$ is not trivial.

Conversely, assume that the stabilizer subgroup $W_{\lambda}^{\circ}$ is not trivial. By Lemma 2.11 there exists a reflection $s \in W_{\lambda}^{\circ}$ such that $s \circ \lambda=\lambda$. It follows from Lemma 2.10 that $\xi^{\lambda}=(-1)^{\ell(s)} \xi^{s \circ \lambda}=-\xi^{\lambda}$. Hence $\xi_{\lambda}=0$.

Given $\lambda \in P^{+}$, let $L(\lambda)$ denote the irreducible highest weight module of $\mathfrak{g}$ with highest weight $\lambda$. The module $L(\lambda)$ admits a weight space decomposition $L(\lambda)=\oplus_{\mu \in \mathfrak{h} *} L_{\mu}$. The character $\operatorname{ch}(L(\lambda))$ of $L(\lambda)$ is defined by

$$
\operatorname{ch}(L(\lambda))=\sum_{\mu \in \mathfrak{h}^{*}}\left(\operatorname{dim} L_{\mu}\right) e^{\mu}
$$

If $\lambda \in P^{+}$, then by [Ka90] we have

$$
\pi^{\lambda}=\operatorname{ch}(L(\lambda)) .
$$

Theorem 1.1 is a consequence of the following result.

Theorem 2.13. Given a Kac-Moody algebra $\mathfrak{g}$, let $P^{+}$denote its set of dominant integral weights and $Q_{\mathrm{im}}^{-}$its negative imaginary root cone. If $f \in \mathcal{G}^{W}(\mathbb{Z}[[q]])$ is such that $\operatorname{supp}(f) \subset Q^{-}$, then there are $c_{\lambda} \in \mathbb{Z}[[q]], \lambda \in P^{+} \cap Q_{\mathrm{im}}^{-}$, such that

$$
f=\sum_{\lambda \in P^{+} \cap Q_{\mathrm{im}}^{-}} c_{\lambda} \operatorname{ch}(L(\lambda)) .
$$

Proof. Since $f$ is supported on $Q^{-}$, we may write the following product as a sum supported on $Q^{-}$:

$$
\Xi=f \cdot \prod_{\alpha \in \Phi^{+}}\left(1-e^{-\alpha}\right)^{m(\alpha)}=\sum_{\beta \in Q^{-}} c_{\beta} e^{\beta} .
$$


As $f$ is invariant under $W$, it follows from Lemma 2.10(1) that

$$
\begin{aligned}
w\left(f \cdot \prod_{\alpha \in \Phi^{+}}\left(1-e^{-\alpha}\right)^{m(\alpha)}\right) & =\sum_{\beta \in Q^{-}} c_{\beta} e^{w \beta} \\
= & (-1)^{\ell(w)} e^{\rho-w \rho} f \cdot \prod_{\alpha \in \Phi^{+}}\left(1-e^{-\alpha}\right)^{m(\alpha)}=\sum_{\gamma \in Q^{-}}(-1)^{\ell(w)} c_{\gamma} e^{\rho-w \rho+\gamma} .
\end{aligned}
$$

Comparing coefficients, we see that for $\beta \in Q^{-}$,

$$
c_{\beta}=(-1)^{\ell(w)} c_{w \circ \beta} .
$$

Moreover, $c_{\beta}=0$ unless $\beta \in Q^{\prime}$, i.e. $\Xi$ is supported on $Q^{\prime}$. If $\lambda+\rho \in P^{+}$and $\lambda \notin P^{+} \cap Q^{-}$for $\lambda \in Q^{-}$, then there exists $\alpha_{i}^{\vee}$ such that $(\lambda+\rho)\left(\alpha_{i}^{\vee}\right)=0$ and $s_{i} \circ \lambda=\lambda$. Thus $\xi^{\lambda}=0$ by Lemma 2.12.

By Lemma 2.11(1) and the above argument, we group the terms of equation (2.21) to get a sum over $P^{+} \cap Q^{-}$, which is the subset of representatives $\lambda$ of the o-action of $W$ on $Q^{\prime}$ such that $\xi^{\lambda} \neq 0$ :

$$
\Xi=\sum_{\beta \in Q^{\prime}} c_{\beta} e^{\beta}=\sum_{\lambda \in P^{+} \cap Q^{-}} c_{\lambda} \xi^{\lambda} .
$$

On the other hand, for $\lambda \in P^{+}$, Weyl's character formula implies

$$
\xi^{\lambda}=\operatorname{ch}(L(\lambda)) \prod_{\alpha \in \Phi^{+}}\left(1-e^{-\alpha}\right)^{m(\alpha)} .
$$

The result follows from combining (2.23) with (2.24), noting that $f$ is in fact supported on $Q_{\mathrm{im}}^{-}$.

Remark 2.14. As mentioned in the introduction, we recover (1.1) as an immediate consequence of Theorem 2.13, since $P^{+} \cap Q_{\mathrm{im}}^{-}=\{0\}$ for finite root systems. In the affine case, we have $P^{+} \cap Q_{\mathrm{im}}^{-}=\mathbb{Z}_{\leq 0} \cdot \delta$ with the minimal positive imaginary root $\delta$, and the theorem shows that the right-hand side of (2.20) only involves imaginary roots.

\section{A formula For the CHARACter COEFficients}

In this section, we derive a formula for the coefficients in the expansion of $\mathcal{M}(q)$ into a sum of characters. We begin with the definition of a function which will play an important role in what follows.

Definition 3.1 ([KL11, KL12]). The function $H: Q^{+} \rightarrow \mathbb{Z}[q]$ is defined by the generating series in $\mathcal{G}(\mathbb{Z}[q])$ :

$$
\sum_{\mu \in Q^{+}} H(\mu ; q) e^{-\mu}=\prod_{\alpha \in \Phi^{+}}\left(1-q e^{-\alpha}\right)^{m(\alpha)},
$$

where $m(\alpha)$ is the multiplicity of $\alpha$. When we do not need to specify $q$, we will frequently write $H(\mu)=H(\mu ; q)$.

Remark 3.2. In [KL11, KL12], the function $H$ was denoted by $H_{\rho}$. See (2-13) in [KL12].

Definition 3.3. Let $\mu \in Q^{+}$, and $\mathscr{P}:=\left\{(\alpha ; i): \alpha \in \Phi^{+}, i=1,2, \ldots, m(\alpha)\right\}$. An admissible partition of $\mu$ is a finite set $\mathfrak{p} \subset \mathscr{P}$ such that $\sum_{(\alpha, i) \in \mathfrak{p}} \alpha=\mu$. Let $\mathcal{P}(\mu)$ be the set of admissible partitions of $\mu$. Given $\mathfrak{p} \in \mathcal{P}(\mu)$, we will refer to an element $(\alpha, i) \in \mathfrak{p}$ as part of $\mathfrak{p}$, and denote the number of parts in $\mathfrak{p}$ by $|\mathfrak{p}|$.

Examples of admissible partitions are given in Appendix A. 
Lemma 3.4. We have

$$
H(\mu)=\sum_{\mathfrak{p} \in \mathcal{P}(\mu)}(-q)^{|\mathfrak{p}|}
$$

Proof. Equation (3.2) follows from expanding the product in equation (3.1) and computing the coefficient of $e^{-\mu}$.

We now prove equation (1.3), which we state below as a theorem for ease of reference.

Theorem 3.5. For $\lambda \in P^{+} \cap Q_{\mathrm{im}}^{-}$, define $d_{\lambda}$ by equation (1.2). Then we have

$$
\chi(q) d_{\lambda}=\sum_{w \in W}(-1)^{\ell(w)} H(-w \circ \lambda) .
$$

Proof. By definition, we have

$$
\begin{aligned}
\mathcal{M}(q) & =\sum_{w \in W} \Delta^{w}=\sum_{w \in W} \prod_{\alpha \in \Phi^{+}} \frac{\left(1-q e^{-w \alpha}\right)^{m(\alpha)}}{\left(1-e^{-w \alpha}\right)^{m(\alpha)}} \\
& =\sum_{w \in W} \frac{\sum_{\mu \in Q^{+}} H(\mu) e^{-w \mu}}{\prod_{\alpha \in \Phi^{+}}\left(1-e^{-w \alpha}\right)^{m(\alpha)}} \\
& =\sum_{\mu \in Q^{-}} H(-\mu) \sum_{w \in W} \frac{e^{w \mu}}{\prod_{\alpha \in \Phi^{+}}\left(1-e^{-w \alpha}\right)^{m(\alpha)}} .
\end{aligned}
$$

Using Lemma 2.10 (1), we deduce that

$$
\mathcal{M}(q)=\frac{1}{\prod_{\alpha \in \Phi^{+}}\left(1-e^{-\alpha}\right)^{m(\alpha)}} \sum_{\mu \in Q^{-}} \sum_{w \in W}(-1)^{\ell(w)} H(-\mu) e^{w \circ \mu} .
$$

As in the proof of Theorem 2.13, put

$$
\Xi:=\mathcal{M}(q) \prod_{\alpha \in \Phi^{+}}\left(1-e^{-\alpha}\right)^{m(\alpha)}=\sum_{\mu \in Q^{-}} \sum_{w \in W}(-1)^{\ell(w)} H(-\mu) e^{w \circ \mu} .
$$

Since $\Xi$ is supported on $Q^{\prime}$, we may rewrite the above double sum as

$$
\Xi=\sum_{\beta \in Q^{\prime}} \sum_{w \in W}(-1)^{\ell(w)} H(-w \circ \beta) e^{\beta} .
$$

The theorem then follows from (2.23).

Example 3.6. Given $w \in W$, write $w=s_{i_{1}} \cdots s_{i_{\ell}}$ as a reduced expression. If $\Phi\left(w^{-1}\right)$ is as defined in equation (2.14), then

$$
\Phi\left(w^{-1}\right)=\left\{\alpha_{i_{1}}, s_{i_{1}}\left(\alpha_{i_{2}}\right), \ldots, s_{i_{1}} \cdots s_{i_{\ell-1}}\left(\alpha_{i_{\ell}}\right)\right\}
$$

and

$$
w \circ 0=\rho-w \rho=\sum_{\alpha \in \Phi\left(w^{-1}\right)} \alpha=\alpha_{i_{1}}+s_{i_{1}}\left(\alpha_{i_{2}}\right)+\cdots+s_{i_{1}} \cdots s_{i_{\ell-1}}\left(\alpha_{i_{\ell}}\right) .
$$

Suppose that

$$
\rho-w \rho=\beta_{1}+\beta_{2}+\cdots+\beta_{k}
$$

for some positive roots $\beta_{1}, \ldots, \beta_{k} \in \Phi^{+}$. Note that we have

$$
s_{i_{1}}(\rho-w \rho) \notin Q^{+} \text {. }
$$


Since $s_{i_{1}}$ keeps $\Phi^{+}$except $\alpha_{i_{1}}$, one of the $\beta_{i}$ 's must be equal to $\alpha_{i_{1}}$. Then $s_{i_{1}}\left(\rho-w \rho-\alpha_{i_{1}}\right)$ is equal to $\rho-w^{\prime} \rho$ where $w^{\prime}=s_{i_{2}} \cdots s_{i_{\ell}}$. Arguing by induction on $\ell(w)$, we deduce that (3.5) is the unique decomposition of $w \circ 0$ into a sum of positive roots. Now it follows from (3.2) that

$$
H(-w \circ 0)=H(\rho-w \rho)=(-q)^{\ell(w)},
$$

and so the formula (3.3) yields

$$
d_{0}=1 \text {. }
$$

Lemma 3.7. For all nonzero $\lambda \in P^{+} \cap Q^{-}$and $w \in W$, the coefficients of $H(-w \circ \lambda)$ sum to zero.

Proof. From [KL12, Lemma 3.18], we have

$$
H(\mu ; 1)= \begin{cases}(-1)^{\ell(w)}, & \text { if } \rho-w \rho=\mu \text { for some } w \in W \\ 0, & \text { otherwise. }\end{cases}
$$

Therefore, it suffices to show that

$$
-w \circ \lambda=-(w(\lambda+\rho)-\rho)=\rho-w(\lambda+\rho) \neq \rho-v \rho
$$

for any $v \in W$. Equation (3.8) is equivalent to $\lambda+\rho \neq w^{-1} v \rho$, and so it is enough to show, for any $v \in W$,

$$
\lambda \neq v \rho-\rho .
$$

If $v=1$ there is nothing to prove. Consider an arbitrary $v \neq 1$, and write $v^{-1}$ as a reduced word $s_{i_{1}} \cdots s_{i_{k}}$. Then we have

$$
\rho\left(v^{-1} \alpha_{i_{k}}^{\vee}\right)<0
$$

and

$$
(v \rho-\rho)\left(\alpha_{i_{k}}^{\vee}\right)=v \rho\left(\alpha_{i_{k}}^{\vee}\right)-\rho\left(\alpha_{i_{k}}^{\vee}\right)=\rho\left(v^{-1} \alpha_{i_{k}}^{\vee}\right)-1 \leq-2 .
$$

Thus $v \rho-\rho \notin P^{+}$. Since $\lambda \in P^{+}$, we have $\lambda \neq v \rho-\rho$.

Definition 3.8. Let $\lambda \in Q^{-}$and

$$
\mathfrak{p}=\left\{\left(\beta_{1} ; m_{1}\right),\left(\beta_{2} ; m_{2}\right), \ldots,\left(\beta_{t} ; m_{t}\right)\right\} \in \mathcal{P}(-\lambda) .
$$

Given $w \in W$, we define

$$
m(\mathfrak{p}, w)=t-2 \times \#\left\{\left(\beta_{i} ; j\right) \in \mathfrak{p}: w \beta_{i}<0\right\} .
$$

With $\mathfrak{p}$ as in equation (3.9), we define

$$
\phi_{i}(\mathfrak{p}):= \begin{cases}\left\{\left(s_{i} \beta_{1} ; m_{1}\right), \ldots,\left(s_{i} \beta_{t} ; m_{t}\right),\left(\alpha_{i} ; 1\right)\right\}, & \text { if } \beta_{j} \neq \alpha_{i} \text { for any } j, \\ \left\{\left(s_{i} \beta_{1} ; m_{1}\right), \ldots,\left(s_{i} \beta_{j-1} ; m_{j-1}\right),\left(s_{i} \beta_{j+1} ; m_{j+1}\right), \ldots,\left(s_{i} \beta_{t} ; m_{t}\right)\right\}, & \text { if } \beta_{j}=\alpha_{i} \text { for some } j .\end{cases}
$$

Since

$$
-s_{i} \circ \lambda=-s_{i}(\lambda+\rho)+\rho=-s_{i}(\lambda)+\alpha_{i},
$$

we see that $\phi_{i}(\mathfrak{p}) \in \mathcal{P}\left(-s_{i} \circ \lambda\right)$. In other words, $\phi_{i}$ defines a map $\mathcal{P}(-\lambda) \rightarrow \mathcal{P}\left(-s_{i} \circ \lambda\right)$. Replacing $\lambda$ with $s_{i} \circ \lambda$, we obtain similarly a map from $\mathcal{P}\left(-s_{i} \circ \lambda\right)$ to $\mathcal{P}(-\lambda)$. One can check that these maps are inverses to each other, and so the map $\phi_{i}$ is a bijection from $\mathcal{P}(-\lambda)$ to $\mathcal{P}\left(-s_{i} \circ \lambda\right)$.

Lemma 3.9. If $\ell\left(w s_{i}\right)=\ell(w)+1$, then

$$
m\left(\phi_{i}(\mathfrak{p}), w\right)=m\left(\mathfrak{p}, w s_{i}\right)+1 .
$$


Proof. Consider $\phi_{i}(\mathfrak{p})=\left\{\left(\beta_{1} ; m_{1}\right), \ldots,\left(\beta_{t} ; m_{t}\right)\right\} \in \mathcal{P}(-\lambda)$. First assume that

$$
\mathfrak{p}=\left\{\left(s_{i} \beta_{1} ; m_{1}\right), \ldots,\left(s_{i} \beta_{t} ; m_{t}\right),\left(\alpha_{i} ; 1\right)\right\} .
$$

By applying $w s_{i}$ to the first components, we get $w \beta_{1}, \ldots, w \beta_{t},-w \alpha_{i}$. Since $-w \alpha_{i}<0$ from the condition $\ell\left(w s_{i}\right)=\ell(w)+1$, we obtain $m\left(\mathfrak{p}, w s_{i}\right)=m\left(\phi_{i}(\mathfrak{p}), w\right)+1-2=m\left(\phi_{i}(\mathfrak{p}), w\right)-1$. Next assume that

$$
\mathfrak{p}=\left\{\left(s_{i} \beta_{1} ; m_{1}\right), \ldots,\left(s_{i} \beta_{j-1} ; m_{j-1}\right),\left(s_{i} \beta_{j+1} ; m_{j+1}\right), \ldots,\left(s_{i} \beta_{t} ; m_{t}\right)\right\} .
$$

In this case, we have $\beta_{j}=\alpha_{i}$, and obtain $m\left(\mathfrak{p}, w s_{i}\right)=m\left(\phi_{i}(\mathfrak{p}), w\right)-1$.

Proposition 3.10. If $\lambda \in Q^{-}$and $w \in W$, then

$$
(-1)^{\ell(w)} H(-w \circ \lambda)=q^{\ell(w)} \sum_{\mathfrak{p} \in \mathcal{P}(-\lambda)}(-q)^{m(\mathfrak{p}, w)} .
$$

Proof. Write $w=s_{i_{1}} s_{i_{2}} \cdots s_{i_{\ell}}$ as a reduced expression. By Lemma 3.9, we have

$$
\begin{aligned}
& H\left(-s_{i_{1}} s_{i_{2}} \cdots s_{i_{\ell}} \circ \lambda\right)=\sum_{\mathfrak{p} \in \mathcal{P}\left(-s_{i_{1}} s_{i_{2}} \cdots s_{i_{\ell}} \circ \lambda\right)}(-q)^{m(\mathfrak{p}, \mathrm{id})} \\
& =\sum_{\mathfrak{p} \in \mathcal{P}\left(-s_{i_{2}} \cdots s_{i_{\ell}} \circ \lambda\right)}(-q)^{m\left(\phi_{s_{i_{1}}}(\mathfrak{p}), \mathrm{id}\right)}=\sum_{\mathfrak{p} \in \mathcal{P}\left(-s_{i_{2}} \cdots s_{i_{\ell}} \circ \lambda\right)}(-q)^{m\left(\mathfrak{p}, s_{i_{1}}\right)+1} \\
& =\sum_{\mathfrak{p} \in \mathcal{P}\left(-s_{i_{3}} \cdots s_{i_{\ell}} \circ \lambda\right)}(-q)^{m\left(\phi_{s_{i_{2}}}(\mathfrak{p}), s_{i_{1}}\right)}=\sum_{\mathfrak{p} \in \mathcal{P}\left(-s_{i_{3}} \cdots s_{i_{\ell}} \circ \lambda\right)}(-q)^{m\left(\mathfrak{p}, s_{i_{1}} s_{i_{2}}\right)+2} \\
& =\cdots=\sum_{\mathfrak{p} \in \mathcal{P}(-\lambda)}(-q)^{m\left(\mathfrak{p}, s_{i_{1}} \cdots s_{i_{\ell}}\right)+\ell},
\end{aligned}
$$

which amounts to the identity (3.11).

\section{Polynomiality}

In this section we prove Theorem 1.2. That is, we show that $d_{\lambda}$ is a polynomial when the Weyl group $W$ of $\mathfrak{g}$ is a universal Coxeter group.

Assume that $W$ be a universal Coxeter group of rank $n \in \mathbb{Z}_{>0}$. By definition, the group $W$ is isomorphic to the free product of $n$-copies of $\mathbb{Z} / 2 \mathbb{Z}$. Denote its generators by $s_{i}, i=1, \ldots, n$. The identity element is the only word of length 0 , and for any $\ell \geq 1$ there are $n(n-1)^{\ell-1}$ words of length $\ell$. We thus compute the Poincaré series of $W$ to be:

$$
\chi(q)=\sum_{w \in W} q^{\ell(w)}=1+n q \sum_{k=0}^{\infty}((n-1) q)^{k}=1+\frac{n q}{1-(n-1) q}=\frac{1+q}{1-(n-1) q} \in \mathbb{Z}[[q]] .
$$

Given $\lambda \in P^{+} \cap Q_{\mathrm{im}}^{-}$, our aim is to establish the polynomiality of

$$
d_{\lambda}=\frac{\sum_{w \in W}(-1)^{\ell(w)} H(w \circ \lambda)}{\chi(q)}=\frac{(1-(n-1) q) \sum_{w \in W}(-1)^{\ell(w)} H(w \circ \lambda)}{1+q} \in \mathbb{Z}[[q]] .
$$

Fix $\lambda \in P^{+} \cap Q_{\text {im }}^{-}$, and define

(4.2) $N=\max \left\{\ell(w)+1:\left(w \alpha_{i} ; j\right)\right.$ is a part of any $\mathfrak{p} \in \mathcal{P}(-\lambda)$ for $1 \leq i \leq n, j \in \mathbb{Z}$ and $\left.w \in W\right\}$. 
Since $W$ is a universal Coxeter group, the number $N$ is well-defined. For the time being, fix an arbitrary element $v \in W$ of length $N$. Let $W_{v}$ be the set of elements in $W$ whose reduced word has $v$ as its rightmost factor. For $\mathfrak{p} \in \mathcal{P}(-\lambda)$, define

$$
m\left(\mathfrak{p}, W_{v}\right)=|\mathfrak{p}|-2 \times \#\left\{\left(\beta_{i} ; j\right) \in \mathfrak{p}: w \beta_{i}<0 \text { for some } w \in W_{v}\right\} .
$$

Write

$$
\sum_{\mathfrak{p} \in \mathcal{P}(-\lambda)}(-q)^{m\left(\mathfrak{p}, W_{v}\right)}=\sum_{k=0}^{r} a_{k} q^{k}
$$

for some $a_{k} \in \mathbb{Z}$ and $r \geq 0$, and define

$$
\begin{aligned}
Q_{v} & :=\sum_{k=0}^{r-1}\left((n-1)^{k} a_{0}+(n-1)^{k-1} a_{1}+(n-1)^{k-2} a_{2}+\cdots+a_{k}\right) q^{k}, \\
A_{v} & :=(n-1)^{r} a_{0}+(n-1)^{r-1} a_{1}+(n-1)^{r-2} a_{2}+\cdots+a_{r} .
\end{aligned}
$$

It follows from (3.11) that

$$
\begin{aligned}
\sum_{w \in W_{v}}(-1)^{\ell(w)} H(-w \circ \lambda) & =\sum_{w \in W_{v}} q^{\ell(w)} \sum_{\mathfrak{p} \in \mathcal{P}(-\lambda)}(-q)^{m(\mathfrak{p}, w)} \\
& =q^{N}\left(Q_{v}+A_{v} q^{r}\left(1+(n-1) q+(n-1)^{2} q^{2}+\cdots\right)\right) \\
& =q^{N}\left(Q_{v}+A_{v} \frac{q^{r}}{1-(n-1) q}\right),
\end{aligned}
$$

and so

$$
\begin{aligned}
\sum_{w \in W}(-1)^{\ell(w)} H(-w \circ \lambda) & \\
& =\sum_{w \in W, \ell(w)<N} q^{\ell(w)} \sum_{\mathfrak{p} \in \mathcal{P}(-\lambda)}(-q)^{m(\mathfrak{p}, w)}+q^{N} \sum_{v \in W, \ell(v)=N}\left(Q_{v}+A_{v} \frac{q^{r}}{1-(n-1) q}\right) .
\end{aligned}
$$

Proposition 4.1. For any $w \in W$, the sum

$$
\sum_{w \in W}(-1)^{\ell(w)} H(-w \circ \lambda)
$$

is divisible by $1+q$.

Proof. Let $N$ be defined as in (4.2), and $v \in W$ be an arbitrary element of length $N$. For any $w \in W$, we have

$$
\sum_{\mathfrak{p} \in \mathcal{P}(-\lambda)} 1^{m(\mathfrak{p}, w)}=|\mathcal{P}(-\lambda)|
$$

Therefore

$$
\sum_{\substack{w \in W \\
\ell(w)<N}}(-1)^{\ell(w)} \sum_{\mathfrak{p} \in \mathcal{P}(-\lambda)} 1^{m(\mathfrak{p}, w)}=|\mathcal{P}(-\lambda)| \sum_{\begin{array}{c}
w \in W \\
\ell(w)<N
\end{array}}(-1)^{\ell(w)} .
$$

Since

$$
\sum_{\substack{w \in W \\ \ell(w)<N}}(-1)^{\ell(w)}=\sum_{k=0}^{N-1}(-1)^{k} \#\{w \in W: \ell(w)=k\},
$$


and

we deduce

$$
\#\{w \in W: \ell(w)=k\}= \begin{cases}1, & k=0 \\ n(n-1)^{k-1}, & k>0\end{cases}
$$

$$
\begin{aligned}
\sum_{\substack{w \in W \\
\ell(w)<N}}(-1)^{\ell(w)} & =1-n+n(n-1)-n(n-1)^{2}+\cdots+(-1)^{N-1} n(n-1)^{N-2} \\
& =1-n\left[1+(-1)(n-1)+(-1)^{2}(n-1)^{2}+\cdots+(-1)^{N-2}(n-1)^{N-2}\right] \\
& =1-n\left[1+(1-n)+(1-n)^{2}+\cdots+(1-n)^{N-2}\right] \\
& =1-n\left(\frac{1-(1-n)^{N-1}}{1-(1-n)}\right)=(1-n)^{N-1} .
\end{aligned}
$$

Combining the above, we see that

$$
\sum_{\substack{w \in W \\ \ell(w)<N}}(-1)^{\ell(w)} \sum_{\mathfrak{p} \in \mathcal{P}(-\lambda)} 1^{m(\mathfrak{p}, w)}=(1-n)^{N-1}|\mathcal{P}(-\lambda)| .
$$

Let $A_{v}$ and $Q_{v}$ be defined as in (4.6) and (4.5), respectively. Then we have

$$
\frac{(-1)^{r}}{n} A_{v}=\frac{(-1)^{r}}{n}\left((n-1)^{r} a_{0}+(n-1)^{r-1} a_{1}+\cdots+a_{r}\right),
$$

and

$$
\begin{aligned}
Q_{v}=a_{0} & \left(1-(n-1)+(n-1)^{2}+\cdots+(-1)^{r-1}(n-1)^{r-1}\right) \\
-a_{1}(1- & \left.(n-1)+(n-1)^{2}+\cdots+(-1)^{r-2}(n-1)^{r-2}\right)+\cdots+(-1)^{r-1} a_{r-1} \\
& =\frac{1-(1-n)^{r}}{n} a_{0}-\frac{1-(1-n)^{r-1}}{n} a_{1}+\cdots+(-1)^{r-2} \frac{1-(1-n)^{2}}{n} a_{r-2}+(-1)^{r-1} a_{r-1},
\end{aligned}
$$

so that

$$
Q_{v}+A_{v} \frac{(-1)^{r}}{n}=\frac{1}{n}\left(a_{0}-a_{1}+a_{2}-a_{3}+\cdots+(-1)^{r} a_{r}\right)=\frac{1}{n}|\mathcal{P}(-\lambda)| .
$$

Evaluating (4.7) at $q=-1$, we get

$$
(1-n)^{N-1}|\mathcal{P}(-\lambda)|+(-1)^{N} n(n-1)^{N-1} \frac{1}{n}|\mathcal{P}(-\lambda)|=0 .
$$

Proof of Theorem 1.2. It follows from (4.7) that

$$
(1-(n-1) q) \sum_{w \in W}(-1)^{\ell(w)} H(-w \circ \lambda)
$$

is a polynomial. By Proposition 4.1, the sum $\sum_{w \in W}(-1)^{\ell(w)} H(-w \circ \lambda)$ is divisible by $1+q$. Thus we see from (4.1) that $d_{\lambda}$ is a polynomial.

Remark 4.2. From [KL12, (3-21)], we know that

$$
H(-w \circ \lambda ;-1)=H(\rho-w(\lambda+\rho) ;-1)=\operatorname{dim} V(\rho)_{w(\lambda+\rho)}=\operatorname{dim} V(\rho)_{\lambda+\rho} .
$$

Taking the alternating sum, we get

$$
\sum_{w \in W}(-1)^{\ell(w)} H(-w \circ \lambda ;-1)=\operatorname{dim} V(\rho)_{\lambda+\rho} \sum_{w \in W}(-1)^{\ell(w)},
$$


which does not converge. In Proposition 4.1, the sum $\sum_{w \in W}(-1)^{\ell(w)} H(-w \circ \lambda ; q)$ is to be interpreted via its analytic continuation given by the rational function in equation (4.7).

\section{Appendix A.}

In this appendix, we consider the explicit example of the Kac-Moody algebra $\mathfrak{g}=\mathcal{H}(3)$ associated to the generalized Cartan matrix

$$
A=\left(\begin{array}{cc}
2 & -3 \\
-3 & 2
\end{array}\right)
$$

The Weyl group $W$ is the universal Coxeter group of rank 2, that is, $W$ is isomorphic to the free product $(\mathbb{Z} / 2 \mathbb{Z}) *(\mathbb{Z} / 2 \mathbb{Z})$. As there are two elements for a given length $\geq 1$, the Poincaré series has the following closed form:

$$
\chi(q)=1+2 q \sum_{\ell=0}^{\infty} q^{\ell}=1+2 q\left(\frac{1}{1-q}\right)=\frac{1+q}{1-q} \in \mathbb{Z}[[q]] .
$$

We denote the simple roots by $\alpha_{1}, \alpha_{2}$ and the simple reflections by $s_{1}, s_{2}$ as before. When $\mathfrak{p}=$ $\left\{\left(\beta_{1} ; m_{1}\right),\left(\beta_{2} ; m_{2}\right), \ldots,\left(\beta_{t} ; m_{t}\right)\right\}$ is an admissible partition, we will sometimes write

$$
\mathfrak{p}=\left(\beta_{1} ; m_{1}\right)+\left(\beta_{2} ; m_{2}\right)+\cdots+\left(\beta_{t} ; m_{t}\right) .
$$

For the root multiplicities of $\mathcal{H}(3)$, we refer the reader to [KaMe].

Example A.1. Consider $\lambda=-2 \alpha_{1}-2 \alpha_{2} \in Q^{-}$. Then there are 4 admissible partitions of $-\lambda$ :

(1) $\left(2 \alpha_{1}+2 \alpha_{2} ; 1\right)$

(2) $\left(\alpha_{1} ; 1\right)+\left(\alpha_{1}+2 \alpha_{2} ; 1\right)$,

(3) $\left(\alpha_{2} ; 1\right)+\left(2 \alpha_{1}+\alpha_{2} ; 1\right)$,

(4) $\left(\alpha_{1} ; 1\right)+\left(\alpha_{2} ; 1\right)+\left(\alpha_{1}+\alpha_{2} ; 1\right)$.

All the roots appearing in the list above have multiplicity 1, and so

$$
H(-\lambda)=H\left(2 \alpha_{1}+2 \alpha_{2}\right)=-q+2 q^{2}-q^{3}=-q(q-1)^{2} .
$$

We calculate

$$
-s_{1} \circ \lambda=-s_{1}(\lambda+\rho)+\rho=5 \alpha_{1}+2 \alpha_{2},
$$

and see that $-s_{1} \circ \lambda$ has 4 admissible partitions:

(1) $\left(5 \alpha_{1}+2 \alpha_{2} ; 1\right)$

(2) $\left(\alpha_{1} ; 1\right)+\left(4 \alpha_{1}+2 \alpha_{2} ; 1\right)$,

(3) $\left(2 \alpha_{1}+\alpha_{2} ; 1\right)+\left(3 \alpha_{1}+\alpha_{2} ; 1\right)$

(4) $\left(\alpha_{1} ; 1\right)+\left(\alpha_{1}+\alpha_{2} ; 1\right)+\left(3 \alpha_{1}+\alpha_{2} ; 1\right)$.

Again, all the roots appearing have multiplicity 1 . We therefore deduce that

$$
H\left(-s_{1} \circ \lambda\right)=-q(q-1)^{2}=H(-\lambda) .
$$

Similarly, we compute

$$
H\left(-s_{2} \circ \lambda\right)=-q(q-1)^{2}=H(-\lambda) .
$$

The circle action of $s_{1} s_{2}$ on $\lambda$ yields

$$
-s_{1} s_{2} \circ \lambda=14 \alpha_{1}+5 \alpha_{2},
$$

which is not a root. Yet again we have 4 admissible partitions, but the lengths are different:

(1) $\left(\alpha_{1} ; 1\right)+\left(13 \alpha_{1}+5 \alpha_{2} ; 1\right)$, 
(2) $\left(\alpha_{1} ; 1\right)+\left(3 \alpha_{1}+\alpha_{2} ; 1\right)+\left(10 \alpha_{1}+4 \alpha_{2} ; 1\right)$,

(3) $\left(\alpha_{1} ; 1\right)+\left(5 \alpha_{1}+2 \alpha_{2} ; 1\right)+\left(8 \alpha_{1}+3 \alpha_{2} ; 1\right)$,

(4) $\left(\alpha_{1} ; 1\right)+\left(2 \alpha_{1}+\alpha_{2} ; 1\right)+\left(3 \alpha_{1}+\alpha_{2} ; 1\right)+\left(8 \alpha_{1}+3 \alpha_{2} ; 1\right)$,

in which all the roots still have multiplicity 1 . It follows that

$$
H\left(-s_{1} s_{2} \circ \lambda\right)=q^{2}(q-1)^{2}=-q H\left(-s_{1} \circ \lambda\right)=-q H(-\lambda) .
$$

One can see that this pattern continues as proved in the previous section to yield

$$
H(-w \circ \lambda)=(-q)^{\ell(w)-1} H(-\lambda), \quad w \in W, w \neq \mathrm{id} .
$$

It follows from equations (3.3) and (A.2) that

$$
\begin{aligned}
\sum_{w \in W}(-1)^{\ell(w)} H(-w \circ \lambda) & =H(-\lambda)+\sum_{w \in W, w \neq \mathrm{id}}(-1)^{\ell(w)}(-q)^{\ell(w)-1} H(-\lambda) \\
& =\left(1+q^{-1}\right) H(-\lambda)-q^{-1} \chi(q) H(-\lambda),
\end{aligned}
$$

and so

$$
d_{-2 \alpha_{1}-2 \alpha_{2}}=\left[\left(1+q^{-1}\right) \frac{1-q}{1+q}-q^{-1}\right] H(-\lambda)=-H(-\lambda)=q(q-1)^{2} .
$$

Example A.2. Let $\lambda=-2 \alpha_{1}-3 \alpha_{2} \in Q^{-}$, which is a root with multiplicity 2 . We have admissible partitions:

(1) $\left(2 \alpha_{1}+3 \alpha_{2}, n\right), n \in\{1,2\}$,

(2) $\left(2 \alpha_{1}+2 \alpha_{2}, 1\right)+\left(\alpha_{2}, 1\right)$,

(3) $\left(\alpha_{1}+3 \alpha_{2}, 1\right)+\left(\alpha_{1}, 1\right)$,

(4) $\left(\alpha_{1}+2 \alpha_{2}, 1\right)+\left(\alpha_{1}+\alpha_{2}, 1\right)$,

(5) $\left(\alpha_{1}+2 \alpha_{2}, 1\right)+\left(\alpha_{1}, 1\right)+\left(\alpha_{2}, 1\right)$.

Therefore

$$
H(-\lambda)=-2 q+3 q^{2}-q^{3}=-q(q-1)(q-2) .
$$

We have

$$
-s_{1} \circ \lambda=8 \alpha_{1}+3 \alpha_{2},
$$

which is a root with multiplicity 1 , and admissible partitions:

(1) $\left(8 \alpha_{1}+3 \alpha_{2}, 1\right)$

(2) $\left(7 \alpha_{1}+3 \alpha_{2}, n\right)+\left(\alpha_{1}, 1\right), n \in\{1,2\}$,

(3) $\left(5 \alpha_{1}+2 \alpha_{2}, 1\right)+\left(3 \alpha_{1}+\alpha_{2}, 1\right)$,

(4) $\left(5 \alpha_{1}+2 \alpha_{2}, 1\right)+\left(2 \alpha_{1}+\alpha_{2}, 1\right)+\left(\alpha_{1}, 1\right)$,

(5) $\left(4 \alpha_{1}+2 \alpha_{2}, 1\right)+\left(3 \alpha_{1}+\alpha_{2}, 1\right)+\left(\alpha_{1}, 1\right)$.

Therefore

$$
H\left(-s_{1} \circ \lambda\right)=-q+3 q^{2}-2 q^{3}=-q(q-1)(2 q-1) .
$$

On the other hand

$$
-s_{2} \circ \lambda=2 \alpha_{1}+4 \alpha_{2},
$$

which is a root of multiplicity 1 , and admissible partitions:

(1) $\left(2 \alpha_{1}+4 \alpha_{2}, 1\right)$

(2) $\left(2 \alpha_{1}+3 \alpha_{2}, n\right)+\left(\alpha_{2}, 1\right), n \in\{1,2\}$,

(3) $\left(\alpha_{1}+3 \alpha_{2}, 1\right)+\left(\alpha_{1}+\alpha_{2}, 1\right)$,

(4) $\left(\alpha_{1}+3 \alpha_{2}, 1\right)+\left(\alpha_{1}, 1\right)+\left(\alpha_{2}, 1\right)$,

(5) $\left(\alpha_{1}+2 \alpha_{2}, 1\right)+\left(\alpha_{1}+\alpha_{2}, 1\right)+\left(\alpha_{2}, 1\right)$. 
Therefore:

$$
H\left(-s_{2} \circ \lambda\right)=-q+3 q^{2}-2 q^{3}
$$

Now

$$
-s_{2} s_{1} \circ \lambda=8 \alpha_{1}+22 \alpha_{2},
$$

which is not a root. We make a list of all admissible partitions:

(1) $\left(8 \alpha_{1}+21 \alpha_{2}, 1\right)+\left(\alpha_{2}, 1\right)$,

(2) $\left(7 \alpha_{1}+18 \alpha_{2}, n_{1}\right)+\left(\alpha_{1}+3 \alpha_{2}, 1\right)+\left(\alpha_{2}, 1\right), n_{1} \in\{1,2\}$,

(3) $\left(5 \alpha_{1}+13 \alpha_{2}, 1\right)+\left(3 \alpha_{1}+8 \alpha_{2}, 1\right)+\left(\alpha_{2}, 1\right)$,

(4) $\left(5 \alpha_{1}+13 \alpha_{2}, 1\right)+\left(2 \alpha_{1}+5 \alpha_{2}, 1\right)+\left(\alpha_{1}+3 \alpha_{2}, 1\right)+\left(\alpha_{2}, 1\right)$,

(5) $\left(4 \alpha_{1}+10 \alpha_{2}, 1\right)+\left(3 \alpha_{1}+8 \alpha_{2}, 1\right)+\left(\alpha_{1}+3 \alpha_{2}, 1\right)+\left(\alpha_{2}, 1\right)$.

It follows that

$$
H\left(-s_{2} s_{1} \circ \lambda\right)=q^{2}-3 q^{2}+2 q^{4}=q^{2}\left(2 q^{2}-3 q+2\right) .
$$

On the other hand,

$$
-s_{1} s_{2} \circ \lambda=11 \alpha_{1}+4 \alpha_{2},
$$

which is not a root, and its admissible partitions are:

(1) $\left(10 \alpha_{1}+4 \alpha_{2}, 1\right)+\left(\alpha_{1}, 1\right)$

(2) $\left(8 \alpha_{1}+3 \alpha_{2}, 1\right)+\left(3 \alpha_{1}+\alpha_{2}, 1\right)$,

(3) $\left(8 \alpha_{1}+3 \alpha_{2}, 1\right)+\left(2 \alpha_{1}+\alpha_{2}, 1\right)+\left(\alpha_{1}, 1\right)$,

(4) $\left(7 \alpha_{1}+3 \alpha_{2}, n\right)+\left(3 \alpha_{1}+\alpha_{2}\right)+\left(\alpha_{1}, 1\right), n \in\{1,2\}$,

(5) $\left(5 \alpha_{1}+2 \alpha_{2}, 1\right)+\left(3 \alpha_{1}+2 \alpha_{2}, 1\right)+\left(2 \alpha_{1}+\alpha_{2}, 1\right)+\left(\alpha_{1}, 1\right)$.

Therefore:

$$
H\left(-s_{1} s_{2} \circ \lambda\right)=2 q^{2}-3 q^{3}+q^{4}=q^{2}(q-1)(q-2) .
$$

Next

$$
-s_{1} s_{2} s_{1} \circ \lambda=59 \alpha_{1}+22 \alpha_{2}
$$

which is not a root. We have

$$
H\left(-s_{1} s_{2} s_{1} \circ \lambda\right)=-q^{3}+3 q^{4}-2 q^{5} .
$$

Also

$$
-s_{2} s_{1} s_{2} \circ \lambda=11 \alpha_{1}+30 \alpha_{2}
$$

and

$$
H\left(-s_{2} s_{1} s_{2} \circ \lambda\right)=-2 q^{3}+3 q^{4}-q^{5} .
$$

We arrange the information above into a table, in which the columns are indexed by $n \in \mathbb{N}$ and the rows are indexed by $w \in W$ (written as reduced words, ordered lexicographically). The entry corresponding to row $w$ and column $n$ is the coefficient of $q^{n}$ in $H(-w \circ \lambda)$. An empty space indicates that the coefficient is zero. There is one additional column, which lists the image $w \circ \lambda$ of $\lambda$ under the circle action by $w$, written in coordinates with respect to the basis $\left\{-\alpha_{1},-\alpha_{2}\right\}$. 


\begin{tabular}{|c||c||c|c|c|c|c|c|}
\hline$w$ & $w \circ \lambda$ & 1 & 2 & 3 & 4 & 5 & $\cdots$ \\
\hline \hline id & $(2,3)$ & -2 & 3 & -1 & & & \\
$s_{1}$ & $(8,3)$ & -1 & 3 & -2 & & & \\
$s_{2}$ & $(2,4)$ & -1 & 3 & -2 & & & \\
$s_{2} s_{1}$ & $(8,22)$ & & 1 & -3 & 2 & & \\
$s_{1} s_{2}$ & $(11,4)$ & & 2 & -3 & 1 & & \\
$s_{1} s_{2} s_{1}$ & $(59,22)$ & & & -1 & 3 & -2 & \\
$s_{2} s_{1} s_{2}$ & $(11,30)$ & & & -2 & 3 & -1 & \\
$\vdots$ & $\vdots$ & & & & $\vdots$ & $\vdots$ & $\ddots$ \\
\hline
\end{tabular}

Observe that the strings $(-2,3,-1)$ and $(-1,3,-2)$ repeat with each iteration, shifting 1 space and switching signs as the word length increases. The coefficient of $q^{n}$ in $\chi(q) d_{\lambda}$ can be calculated by taking the sum of the entries in a column multiplied by $(-1)^{\ell(w)}$. We see that

$$
d_{-2 \alpha_{1}-3 \alpha_{2}}=0 \text {. }
$$

Example A.3. Consider $\lambda=-3 \alpha_{1}-3 \alpha_{2} \in Q^{-}$. There are 12 admissible partitions of $-\lambda$ :

(1) $\left(3 \alpha_{1}+3 \alpha_{2} ; 1\right)$

(2) $\left(3 \alpha_{1}+3 \alpha_{2} ; 2\right)$

(3) $\left(3 \alpha_{1}+3 \alpha_{2} ; 3\right)$

(4) $\left(3 \alpha_{1}+2 \alpha_{2} ; 1\right)+\left(\alpha_{2} ; 1\right)$

(5) $\left(3 \alpha_{1}+2 \alpha_{2} ; 2\right)+\left(\alpha_{2} ; 1\right)$

(6) $\left(2 \alpha_{1}+3 \alpha_{2} ; 1\right)+\left(\alpha_{1} ; 1\right)$

(7) $\left(2 \alpha_{1}+3 \alpha_{2} ; 2\right)+\left(\alpha_{1} ; 1\right)$

(8) $\left(2 \alpha_{1}+2 \alpha_{2} ; 1\right)+\left(\alpha_{1}+\alpha_{2} ; 1\right)$,

(9) $\left(2 \alpha_{1}+\alpha_{2} ; 1\right)+\left(\alpha_{1}+2 \alpha_{2} ; 1\right)$,

(10) $\left(\alpha_{1} ; 1\right)+\left(\alpha_{1}+\alpha_{2} ; 1\right)+\left(\alpha_{1}+2 \alpha_{2} ; 1\right)$,

(11) $\left(\alpha_{2} ; 1\right)+\left(\alpha_{1}+\alpha_{2} ; 1\right)+\left(2 \alpha_{1}+\alpha_{2} ; 1\right)$,

(12) $\left(\alpha_{1} ; 1\right)+\left(\alpha_{2} ; 1\right)+\left(2 \alpha_{1}+2 \alpha_{2} ; 1\right)$.

Note that $m\left(3 \alpha_{1}+3 \alpha_{2}\right)=3, m\left(2 \alpha_{1}+3 \alpha_{2}\right)=m\left(3 \alpha_{1}+2 \alpha_{2}\right)=2$, and the other roots each have multiplicity 1 . We conclude

$$
H(-\lambda)=H\left(3 \alpha_{1}+3 \alpha_{2}\right)=-3 q+6 q^{2}-3 q^{3}=-3 q(q-1)^{2} .
$$

We continue to obtain the following table.

\begin{tabular}{|c||c||c|c|c|c|c|c|c|}
\hline$w$ & $w \circ \lambda$ & 1 & 2 & 3 & 4 & 5 & 6 & $\cdots$ \\
\hline \hline id & $(3,3)$ & -3 & 6 & -3 & & & & \\
$s_{1}$ & $(7,3)$ & -2 & 5 & -4 & 1 & & & \\
$s_{2}$ & $(3,7)$ & -2 & 5 & -4 & 1 & & & \\
$s_{1} s_{2}$ & $(19,7)$ & & 2 & -5 & 4 & -1 & & \\
$s_{2} s_{1}$ & $(7,19)$ & & 2 & -5 & 4 & -1 & & \\
$s_{1} s_{2} s_{1}$ & $(19,51)$ & & & -2 & 5 & -4 & 1 & \\
$s_{2} s_{1} s_{2}$ & $(51,19)$ & & & -2 & 5 & -4 & 1 & \\
$\vdots$ & $\vdots$ & & & & $\vdots$ & $\vdots$ & $\vdots$ & $\ddots$ \\
\hline
\end{tabular}

Observe that the string $(-2,5,-4,1)$ repeats with each iteration, shifting 1 space and switching signs as the word length increases. Since $-2+5-4+1=0$, the coefficient of $q^{n}$ is 0 for $n \geq 4$. As 
it happens, the coefficient of $q^{2}$ is 0 too. We conclude

$$
d_{-3 \alpha_{1}-3 \alpha_{2}}=\frac{-q^{3}+q}{\chi(q)}=q(q-1)^{2} .
$$

Example A.4. Let $\lambda=-3 \alpha_{1}-4 \alpha_{2} \in Q^{-}$. We produce a table similar to that in Example A.3

\begin{tabular}{|c||c||c|c|c|c|c|c|c|c|}
\hline$w$ & $w \circ \lambda$ & 1 & 2 & 3 & 4 & 5 & 6 & 7 & $\cdots$ \\
\hline \hline id & $(3,4)$ & -4 & 8 & -5 & 1 & & & & \\
$s_{1}$ & $(10,4)$ & -1 & 7 & -8 & 2 & & & & \\
$s_{2}$ & $(3,6)$ & -3 & 8 & -6 & 1 & & & & \\
$s_{2} s_{1}$ & $(10,27)$ & & 1 & -7 & 8 & -2 & & & \\
$s_{1} s_{2}$ & $(16,6)$ & & 4 & -9 & 5 & & & & \\
$s_{1} s_{2} s_{1}$ & $(72,27)$ & & & -1 & 7 & -8 & 2 & & \\
$s_{2} s_{1} s_{2}$ & $(16,43)$ & & & -4 & 9 & -5 & & & \\
$s_{1} s_{2} s_{1} s_{2}$ & $(72,190)$ & & & & 1 & -7 & 8 & -2 & \\
$s_{2} s_{1} s_{2} s_{1}$ & $(114,43)$ & & & & 4 & -9 & 5 & & \\
$\vdots$ & $\vdots$ & & & & & $\vdots$ & $\vdots$ & $\vdots$ & $\ddots$ \\
\hline
\end{tabular}

This time, the strings $(1,-7,8,-2)$ and $(4,-9,5)$ alternate. Note that both strings sum to zero. We see that for $n \geq 5$, the coefficient of $q^{n}$ in $d_{\lambda}$ is 0 . The coefficients of $q$ and $q^{4}$ are also 0 . Altogether we obtain

$$
d_{-3 \alpha_{1}-4 \alpha_{2}}=\frac{-2 q^{2}(1+q)}{\chi(q)}=2 q^{2}(q-1) .
$$

Example A.5. We may compute other $d_{\lambda}$ 's in a similar way. In the following table, the entry in the space $(m, n)$ is the polynomial $d_{\lambda}$ for $\lambda=-m \alpha_{1}-(m+n) \alpha_{2} \in P^{+}$. From symmetry in $\mathcal{H}(3)$, we have $d_{-m \alpha_{1}-(m+n) \alpha_{2}}=d_{-(m+n) \alpha_{1}-m \alpha_{2}}$.

\begin{tabular}{|c||c|c|c|c|c|}
\hline & 0 & 1 & 2 & 3 & 4 \\
\hline \hline 0 & 1 & $-q(q-1)$ & $q(q-1)^{2}$ & $q(q-1)^{2}$ & $2 q(q-1)^{2}$ \\
\hline 1 & & & 0 & $2 q^{2}(q-1)$ & $-q^{2}(q-1)(q-4)$ \\
\hline 2 & & & & & $-q(q-1)^{2}\left(q^{2}+q-1\right)$ \\
\hline
\end{tabular}

We also have

$$
d_{-5 \alpha_{1}-5 \alpha_{2}}=-q(q-1)\left(q^{3}+3 q^{2}-7 q+2\right) .
$$

\section{REFERENCES}

[BPGR16] N. Bardy-Panse, S. Gaussent, and G. Rousseau, Iwahori-Hecke algebras for Kac-Moody groups over local fields, Pacific J. Math. 285 (2016), no. 1, 1-61.

[BPGR19] _ Macdonald's formula for Kac-Moody groups over local fields, Proc. Lond. Math. Soc 119 (2019), no. $1,135-175$.

[BFK] A. Braverman, M. Finkelberg and D. Kazhdan, Affine Gindikin-Karpelevich formula via Uhlenbeck spaces, Contributions in analytic and algebraic number theory, Springer Proc. Math. 9 (2012), 17-29.

[BGKP] A. Braverman, H. Garland, D. Kazhdan and M. Patnaik, An affine Gindikin-Karplevich formula, Contemp. Math. 610 (2014), 43-64.

[BKP] A. Braverman, D. Kazhdan and M. Patnaik, Iwahori-Hecke algebras for p-adic loop groups, Invent. Math. 204 (2016), 347-442.

[Bu12] D. Bump, Introduction: multiple Dirichlet series, Multiple Dirichlet series, L-functions and automorphic forms, Progr. Math. 300 (2012), 1-36.

[Ch95] I. Cherednik, Double affine Hecke algebras and Macdonald's conjectures, Ann. Math. (2) 141 (1995), no. 1, $191-216$. 
[GR14] S. Gaussent and G. Rousseau, Spherical Hecke algebras for Kac-Moody groups over local fields, Ann. Math. (2) 180 (2014), no. 3, 1051-1087.

[Ka90] V. G. Kac, Infinite dimensional Lie algebras, Cambridge University Press, 1990.

[KaMe] S.-J. Kang and D. J. Melville, Rank 2 symmetric hyperbolic Kac-Moody algebras, Nagoya Math. J. 140 (1995), 41-75.

[KL11] H. Kim and K.-H. Lee, Representation theory of p-adic groups and canonical bases, Adv. Math. 227 (2011), no. 2, 945-961.

[KL12] — Quantum affine algebras, canonical bases and q-deformation of arithmetical functions, Pacific J. Math. 255 (2012), no. 2, 393-415.

[MPW] D. Muthiah, A. Puskas and I. Whitehead, Correction factors for Kac-Moody groups and t-deformed root multiplicities, Math. Z. 296 (2020), 127-145.

[M72] I.G. Macdonald, The Poincaré series of a Coxeter group, Math. Ann. 199 (1972), 161-174.

[M82] _ Some conjectures for root systems, SIAM J. Math. Anal. 13 (1982), no. 6, 988-1007.

[M03] , A formal identity for affine root systems, Lie groups and symmetric spaces, Amer. Math. Soc. Transl. Ser. 2, vol. 210 (2003), 195-211.

[St68] R. Steinberg, Endomorphisms of linear algebraic groups, Memoirs of the Amer. Math. Soc. 80, 1968.

Department of Mathematics, University of Connecticut, Storrs, CT 06269, U.S.A.

Email address: khlee@math.uconn.edu

School of Mathematical Sciences, Zhejiang University, Hangzhou 310027, P.R. China

Email address: maliu@zju.edu.cn

Mathematical Institute, University of Oxford, Andrew Wiles Building, Oxford, OX2 6GG, U.K.

Email address: Thomas.Oliver@maths.ox.ac.uk 